\title{
Modeling the Spread of an Obesity Intervention through a Social Network
}

\author{
Mahboobeh Ghesmaty Sangachin, B.S.; Mohammadreza Samadi, M.S.; \\ Lora A. Cavuoto, Ph.D.* \\ Department of Industrial and Systems Engineering, University at Buffalo, \\ Buffalo, NY, USA
}

Submitted December 2013. Accepted for publication April 2014.

\begin{abstract}
With the growing prevalence of obesity and the public health implications, it is critical to develop and evaluate potential interventions. One approach is to investigate the spread of positive health outcomes through a social network. We employ the ground concepts of spread maximization problem and adapt it to best reflect the dynamics of a weight loss intervention. A diffusion model is then employed for the propagation of weight loss effect throughout the network of obese and overweight individuals. This diffusion model integrates both personal attributes and networkrelated attributes of an individual while engaged in a weight loss program. Simulation findings suggest that choosing initial agents based on individual attributes, including ability to lose weight, body mass index, and threshold, produced the highest total weight loss in the network. Greedy algorithm was also applied to choose the most effective subset of initial seeds.
\end{abstract}

Keywords: weight loss, obesity, diffusion model, health-related behavior, social network

\section{INTRODUCTION}

According to the data from the National Health and Nutrition Examination Survey, more than one-third of U.S. adults and almost $17 \%$ of youth were reported obese in 2009-2010 [1,2]. Both overweight and obesity are known to be significantly associated with diabetes, high blood pressure, high cholesterol levels, asthma, arthritis, fair or poor health status and other chronic diseases [3]. Studies also suggest that obesity is more strongly associated with increased health care and medication spending than smoking or alcohol use [4]. Obesity is estimated to increase health care costs $36 \%$ and medications costs $77 \%$ above the costs of being in the normal weight range [5]. With this added burden, it is essential to seek methods to address the issue.

With the expansion of communication technology, individuals have become more connected, particularly through social networks. The power of teams and peers on an individual's behavior has largely been investigated [6-8]. Physical activity which is an

*Corresponding author: Lora A. Cavuoto, $\mathrm{PhD}$, Department of Industrial and Systems Engineering, University at Buffalo, 324 Bell Hall, Buffalo, NY 14226. Phone: (716) 645-4696. Email: loracavu@ buffalo.edu. Other authors: mghesmat@buffalo.edu; msamadi@buffalo.edu. 
important component of many weight loss programs is also shown to be influenced by peers' level of activity. It is reported that team members influence individual activity outcomes in team-based statewide campaigns [9]. The results of a survey among schoolaged children also suggest that friendship ties play a critical role in setting physical activity patterns in children [10]. The activity level of immediate social network during a 12-week afterschool program is shown to largely affect the child's physical activity level. Along with this, a shared environment is not a key factor influencing adolescents' sports playing behavior, which highlights the social network influence [11].

The role of peer influence has also been investigated in the spread of obesity. Analysis of a densely interconnected social network of about 12,000 participants in the Framingham Heart Study (assessed from 1971 to 2003) revealed that an individual's chances of becoming obese increased by $57 \%$ if he or she had a friend who became obese. If one spouse became obese, the likelihood that the other spouse would become obese increased by $37 \%$ [12]. In examining a weight loss intervention, a study found that a majority of study participants with a social contact who also participated in a similar weight loss program reported that their social contact's participation played a key role in their decision to participate in the program [13]. The literature suggests that among young adults, having obese friends trying to lose weight has a positive impact on an individual's willingness to lose weight [14]. How people are connected to each other is also found to impact the efficacy of health interventions, and that the structure of social networks impacts how health messages are transferred among individuals and how people can influence certain aspects of their peers' behavior [15]. Yet the relationship between acquiring knowledge of health issues and practicing the positive behaviour is unclear [15]. Although some recent work [16] has taken advantage of social network concepts to investigate the influence of people on each other's healthrelated behavior and preferences, there remains a need to assess techniques in social network analysis that can enhance the efficacy of network-based interventions. Furthermore, in these previous studies, either network-related attributes or individual traits have been taken into account, separately. There remains a need for an integrated investigation that considers both personal traits and social aspects in determining the likelihood of success in participating in such programs.

In order to model the spread of an obesity intervention through a population, the influence maximization model in social networks can be applied. Individuals in a social network can be modeled as a graph in which the nodes represent the individuals and the edges represent the relationship between them. For this model, a key assumption is that activated individuals exchange their attitudes towards a product or program, whether positive or negative, to their connected neighbors. Under certain conditions, this attitude can continue to spread beyond the immediately connected neighbors to their own linked neighbors. Thus, the number of people being influenced increases and the effect spreads through the network. Based on the degree of nodes (number of its connected neighbors) and other network related characteristics, different sets of initially activated nodes can lead to diverse propagation patterns. The goal of the influence maximization problem is to identify a set of influential nodes in a network to maximize the propagation of the desired influence among nodes. 
The objective of this work is to assess the diffusion of weight loss throughout a network of obese and overweight individuals using a model of the spread of effect with realistic parameters. Although previous studies have shown that friends, siblings or spouses affect each other, no studies have shown how people would be influenced by the conglomerate of such effects over time. Unlike some previous studies, the presented model can show the aggregated effects on an individual when the surrounding people are activated and propagating the weight loss effect. Individuals begin trying to lose weight upon receiving enough effect from their network connections to cross a threshold, and on the other hand, also become discouraged by their connections who fail to lose weight. We propose a model that integrates both personal attributes that lead to adopting a certain behavior and network related attributes of an individual. This diffusion model can be used to decide whom to choose as initial participants of a weight loss program in order to maximize its effectiveness.

\section{METHODS}

\subsection{Model}

Although several diffusion models have been proposed for the spread of ideas in social networks, the spread of weight loss has some unique traits. When applying an influence maximization model to promote a new product in a social network, the goal is to motivate people to purchase the product, with the goal of maximizing the total revenue made from the single decision of purchasing a product [17]. In contrast, to develop a diffusion model for weight loss, both the decision to participate in the program and the individual's ability to lose weight are important factors. Making the decision to lose weight does not necessarily increase the total weight loss in the network and an unsuccessful participant does not increase the total network weight loss while spreading negative feedback about the program to other nodes. We introduce a diffusion model of the propagation of weight loss through a network of connected overweight and obese individuals, that accounts for different behavioral characteristics and inter-individual relationships.

The social network is modeled as an undirected graph $G=(N, E)$ where $N$ represents the set of nodes in the network and $E$ represents the set of bidirected relationships between nodes. Bidirected relationships reflect the nature of the relationships between people in a natural social setting. Social ties between people can have different categories such as kinship and friendship, and the connections can have different weights based on the strength of relationship. This difference is shown by assigning different weights to the edges, as shown in Figure 1. All nodes in the network are individuals who are obese and are potentially willing to participate in a weight loss program. Christakis and Fowler [12] showed that obese individuals in a social network tend to cluster during network formation; therefore, once the network is stabilized, there is potential to have subnetworks containing predominantly overweight and obese individuals.

Influence can be diffused through the network in multiple fashions. Particular diffusion models include the Independent Cascade and Linear Threshold models introduced by Kempe et al. [17]. These models provide general frameworks that define how influence spreads in a social network based on customization to the specific application. With the Independent Cascade model, each activated node has a one-time 


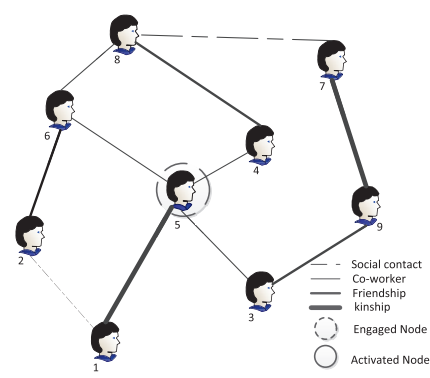

(a)

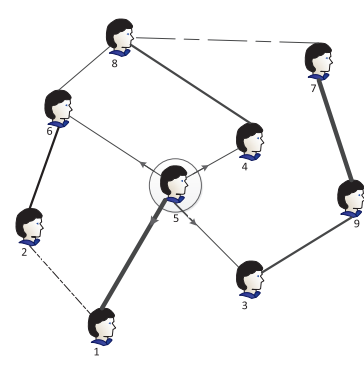

(b)

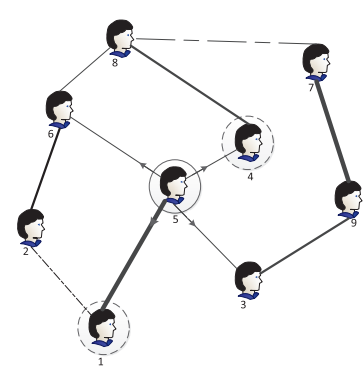

(c)

Figure 1. Graph $\mathrm{G}$ representing the baseline network where (a) Node 5 becomes engaged in the program as an initial seed. (b) Node 5 is activated and has lost weight; therefore it sends out weight loss effect to its neighbors. (c) After perceiving the effect from node 5, nodes 1 and 4's willingness to participate in the program surpass their threshold values and become engaged in the program. Node 5 continues to lose weight, depending on its ability, until attaining a successful weight loss level or reaching normal weight, or ceasing participation due to lack of perceived effectiveness of the intervention.

chance to activate its neighbors. A probability value is assigned to the edges, representing the probability of success for tail to activate the head of the edge. In the Linear Threshold model, every node has a threshold value and every edge has a weight. A node gets activated whenever the summation of incoming weights, from activated neighbors, is greater than its threshold value. In this paper, a Linear Threshold-based model is developed to formulate the spread of weight loss effect in social networks. This model requires two main parameters: threshold value and weight of edge. Threshold value is an individual-based attribute, specifying the resistance of users against participation in a weight loss program. Weight of edge, on the other hand, is an edge-based attribute which determines the strength of the influence flowing between two nodes. We assign the threshold values using the threshold distribution in the network. Edge weight is derived as a function of the strength of relationship between two nodes and the magnitude of lost weight for the sender of the influence.

Throughout the intervention period, one year in this case, each node can take on one of five states: inactive, engaged, active, dismissed, or successful. Initially, a node begins inactive, not participating in the program and thus not sharing any effect with others. At this point, the individual collects effect sent from any activated network neighbors. When the effect crosses the threshold for engagement, the node becomes engaged and commits to the weight loss program, but cannot yet spread any effect. Once an engaged node begins to lose weight, after a time interval of one month, he/she becomes active. An active node can thus send out either positive or negative effects based on weight loss 
success in the program. A dismissed node is the one that has been activated but has not reached the success threshold in losing weight and therefore leaves the program. In practice, these individuals should not be neglected, but may need to be targeted with a different intervention that suits them better. Due to their lack of success, this node sends negative feedback to the connected neighbors. The magnitude of this negative effect is proportional to the weight loss failure. Successful nodes, on the other hand, are those who are engaged and then activated in the program and successfully lose enough weight to no longer be considered overweight or obese. These nodes stay in the network and spread a positive effect while they are obese. Once they reach normal weight, they are excluded from the list of potential future seeds, but not from the intervention. Weight loss maintenance is the second phase of a successful weight loss intervention, and can be modelled separately. We assume that since these individuals have been successful and have transferred their influence to their peers while losing weight, they no longer propagate the effect as they remain in the program.

At the beginning of the process, 4 initial values are assigned to each node $v \in N$ : initial weight $\left(w_{i}\right)$, threshold value $\left(\sigma_{i}\right)$, willingness value $\left(L_{i}\right)$ and ability value $\left(B_{i}\right)$. Initial body weight is a constant for each node showing the weight (in kilograms) of the node at time $(t)=0$. We use BMI (body mass index) as an indicator of obesity; however, since an individual's height is assumed to remain constant, the initial value of interest is body weight. Initial willingness value shows the eagerness of node $v$ at $t=0$ to lose weight. The threshold value is a limen showing the minimum willingness value a node needs to reach in order to engage in the program. It is shown that individuals have different attitude toward receiving advice to lose weight [18]. Ability reflects the capacity of the node $v$ at $t=0$ to lose weight if engaged in the program.

When node $v \in N$ is first engaged at time $t$, the individual has achieved enough motivation to participate in weight loss program, having crossed the threshold value (see Figure 1a). For the next time step, the individual is engaged and begins to make an effort to lose weight. At the end of the engagement period, the node becomes activated either by positive or negative activation. A node is considered successfully engaged when he/she loses weight at a rate greater than or equal to the success weight loss rate $(\alpha)$. $\alpha$ represents the percentage of weight that needs to be lost at each time interval in order to be successful based on previously studied tests of perceived success in weight loss. Previous studies have shown that losing and maintaining a weight loss of $10 \%$ can decrease many of the negative consequences of obesity [19]. We use this finding to set a baseline for judging how successful individuals are in losing weight. Although this rate is typically calculated at the end of any weight loss program, it is also calculated at certain time intervals during the intervention to track individual's progress over time. Once activated, a node keeps record of the amount of lost weight during all time intervals. This record helps in calculating the value of the effect it will send out to its connected neighbors. In practical application of a weight loss program, an additional factor is an individual's ability to lose weight, which decreases with time [20]. The magnitude of this decrease can be calculated based on equations provided by Hall et al. [20] and Hall [21]. 
At a particular time $t$, the effect value is calculated by adding the effect generated in the current period to the value of the effects from previous intervals discounted by rate $\beta$. $\beta$ represents a decay factor based on the assumption that individuals do not remember the entire previously received effects leading to an updating of the value at each time interval. Considering all abovementioned variables and constants, the effects are calculated as below. For each node $v \in N$, let $e_{t}(v)$ be the value of the effect created at time $t$ by node $v$ and $e_{\text {out }}(v)$, the value of the effect it sends out. $e_{p s r} t(S)$ is the effect node $s$ receives from its activated neighbor. For $t \geq 1$, for all activated nodes, $e_{\text {out } t}(v)=$ $e_{t}(v)+(1-\beta) e_{\text {out } t-1}(v)$ and $e_{\text {out } 0}(v)=0$. Each node $s \subseteq I_{t}(v)$ perceives the effect that node $v$ has sent out as $e_{p s r}(S)=e_{\text {out } t}(v)^{*} E W_{v s}$, with $E W_{v s}$ being the weight of the edge between node $v$ and node $s$. The variable values are updated as following:

$\forall v \in N:$

$$
\begin{gathered}
W_{t v}=\left(1-\left(\frac{B_{t v}}{100}\right)\right) \times W_{t-1 v} \\
\text { Weight loss } t(v)=\left(W_{t v}-W_{t-1 v}\right) \\
e_{t}(v)=B_{t v}-\alpha \\
e_{\text {out }}(v)=e_{t}(v)+(1-\beta) e_{\text {out } t-1}(v) \\
B_{t v}=B_{t-1 v}-\left(\delta \times \text { Weight } \operatorname{loss}_{t}(v)\right)
\end{gathered}
$$

$\forall s \subseteq I_{t}(v):$

$$
\begin{gathered}
e_{\text {pert }}(s)=e_{\text {out } t}(v) * E W_{v s} \\
L_{t s}=L_{t-1 s}+e_{\text {pert }}(s)
\end{gathered}
$$

The dynamics of our model can be explained as follows. Discrete time steps $t=0,1$, $2,3, \ldots, T$ are defined to model the dynamic change in the network state. For each node, $v \in N$, let $I_{t}(v), E_{t}(v)$ and $A_{t}(v)$ represent sets of neighbors of $v$, which are inactive, engaged, or activated, respectively, at the beginning of time period $t$. Each node $w \subseteq$ $A_{t}(v)$ sends effect $e_{\text {out } t}(w)$ toward $v \subseteq I_{t}$. The magnitude of $e_{\text {out }} t(w)$ depends on the amount of weight lost by $w$. For each node $w \subseteq A_{t}(v)$, it is decided whether it has 
reached the defined weight loss success rate in the current time interval. If successful, its ability value updates and if not, the node is dismissed from the program. The current BMI status for node $w$ is also checked so that individuals with a BMI $<25 \mathrm{~kg} / \mathrm{m}^{2}$ successfully leave the program and are labeled as successful nodes. Node $v$ accumulates the received effects from all connected neighbors and updates its current willingness value. At the end of each time interval, node $v$ compares its willingness with its threshold value. Node $v$ will be engaged in the program as soon as its willingness exceeds its threshold. The diffusion process can be terminated either after a predefined number of steps $(T)$ or after a preset goal is achieved.

For influence maximization problems, the objective function is traditionally defined as maximizing the number of activated nodes at the end of the diffusion process. In this weight loss model, both the number of activated nodes and the total amount of weight lost over the network are of interest. Participation of nodes in the weight loss program does not provide any positive result on its own. There can be different objectives for a weight loss program [22]. In studies of weight loss intervention effectiveness [23], mean change in weight from baseline has been used as the main effectiveness metric. Since we are also interested in cumulative results in the network, total weight loss is another metric that we selected. Furthermore, the number of individuals who successfully reach a normal weight state is clinically important to consider. Total lost weight at the end of diffusion process is denoted by $\Gamma_{G}(S)$ where $G$ is the obesity graph and $S$ is the set of seeds at the beginning of the process. Initial seeds may be targeted with more advertisement and marketing to encourage them to take part in the program. The ultimate goal remains to influence as many nodes as possible and the initial seeds do not receive any health-related benefit that is not attainable for other nodes. Usually, the size of $S$ is a predefined number, i.e., $k$, and influence maximization scheme tries to find the optimal seed set $S^{*}$ that maximizes the total lost weight in the network over all time steps, i.e., $S^{*} \in \operatorname{argmax}_{S} \subseteq N$, $\left|S_{p}\right|=k{ }_{G}(S)$.

\subsection{Conceptual Justification of the Model}

In modeling the potential effectiveness of an obesity intervention, it is important to match realistic conditions as best as possible. The proposed model was developed with this intention and serves to investigate a real social network of obese and overweight people. The literature suggests that among young adults, having obese friends trying to lose weight has a positive impact on an individual's willingness to lose weight [14]. Therefore, we assume that the same holds for individuals across age groups. Adapting the techniques used in viral marketing and technology adoption [24], we suppose that if an obese or overweight individual is surrounded by other obese or overweight individuals who are trying to lose weight, there is a greater chance for him/her to become willing to participate in a weight loss program. Although online social networks such as Facebook do not resemble offline ones in every aspect, network data can be extracted from an online social network. We also considered the network graph to be weighted, since in reality, different bonds influence in various ways. We include this effect by assigning weights from 1 to 4 to the edges in our obesity graph and account for the actual differences in value while calculating the received effect by each node. 
It was assumed that the behavior of individuals who are overweight or obese differs from lean individuals [25]. Giabbanelli et al. [26] discuss the influence of environment to be as important as social influences; however, they point out that micro-level structural properties can be the main factor in formation of a cohesive population. We use this finding to set our assumption that external elements do not contribute to the outcome as much as the effects produced by nodes inside the network. Consequently, we focus on the node's attributes rather than network attributes. Although the model focuses on interactions between obese nodes, we consider the effect of being surrounded by lean nodes on obese objects. This effect manifests in the initial willingness value assigned to nodes. A certain node is assumed to be more willing to lose weight if it has more lean connecting neighbors around it. Willingness is positively correlated with the social pressure someone feels to adopt a certain behavior to coincide with social norms [14].

An engagement period is a requirement as one of the node states in our model. The engagement period represents the time a node needs to build up some effect. Since weight loss is the behavior of interest, unlike other influence models, it takes time for the node to prepare some level of effect. The duration of the engagement period can be adjusted according to needs without any impact on the model dynamics. We also assumed that the program effect exceeds the transferred effect between two individuals; therefore, an activated node does not receive influence from other activated nodes. In an attempt to model the natural behavior of forgetfulness, previous effects are discounted at a consistent rate $\beta$. Newer information is valued greater than previously received data. When $\beta=0$, only the most recently received effect is considered. On the other hand, $\beta=1$ indicates no forgetfulness. $\beta$ can vary with regard to the actual duration of time steps in the model and the characteristics of the population studied; however, it does not contribute to the structure of the model. Negativity bias presents another concern with human behavior. People tend to care more about the negative effects they receive [27]. In our case, this translates to an easier ability of contacts to discourage rather than motivate. Our model has the potential to consider such a bias.

\subsection{Selection of Initial Seeds}

With the proposed model, the objective was to study the propagation of weight loss through a diffusion model and to identify potential sets of initial seeds, although not necessarily the optimal subset of initial seeds. After development of the model, we tested six different scenarios for selecting the initial seeds. These scenarios come from metrics of the social network (network-based) and variables of individual characteristics related to the efficacy of a weight loss intervention (individual-based). For each scenario, the top $10 \%$ of nodes meeting the criteria were selected as the seeds. The number of initial seeds is usually limited by the intervention budget and does not affect the dynamics of the model.

The network-based scenarios we tested were the following:

Scenario 1: Nodes with highest degree. Degree of a node in a network represents the number of its direct connections. El-Sayed et al. [28] showed that this scenario may not lead to significant difference in program outcome; however, since the current model introduces a new method to quantify the weight loss effect in the network, this scenario was tested. 
Scenario 2: Nodes with highest betweenness centrality. A node's betweenness centrality represents the number of all shortest paths that pass from a node. A node with high betweenness centrality has great influence on the effect flow in the network.

Scenario 3: Nodes with highest closeness centrality. Closeness, as opposed to farness, shows how close a node is to all the other nodes in the network. The total distance to all the other nodes is relatively small for nodes with high closeness centrality; thus they can access to other nodes more quickly.

The individual-based scenarios we tested were the following:

Scenario 4: Nodes with highest ability to lose weight. These nodes are capable of losing more weight than others; therefore, in theory if chosen as seeds, they can generate a greater effect and propagate it.

Scenario 5: Nodes with highest BMI. Individuals with the highest BMI, if selected as seeds, can stay longer in the network and continue to propagate the weight loss effect.

Scenario 6: Nodes with highest initial value of threshold - willingness (requiring the most program effort for engagement). If not selected as nodes, these individuals would need to receive greater effects from their neighbors to become activated; therefore, selecting them as initial seeds can potentially increase the number of participating nodes by the end of the program.

\section{RESULTS AND DISCUSSION}

\subsection{Simulation Characteristics}

Simulation analysis was completed using social media network data from the Stanford University dataset collection, in which nodes are Facebook friends. One of the ten available networks in this dataset was selected [29]. This dataset was publicly available and any personal information about human participants, such as name, age, and gender, had been previously removed from the dataset. The network contains 1034 nodes and has a density of 0.10 with an average degree of nodes of 103.5. All experiments have been done on a desktop with Intel(R)Core(TM)i3 3.3GHz processor, 8GB RAM and 64 bit operating system, and all simulation processes have been coded in MATLAB R2013a.

As mentioned above, a weight loss of $10 \%$ per year is considered attainable and beneficial for overweight or obese individuals [30]. In addition, a weight loss of 5\% is shown to significantly reduce disorders related to obesity, including high cholesterol, high blood pressure, and cardiovascular disease [31]. Considering the reported outcomes of commercial weight loss programs [32], we set $\alpha$ at 5\% a year. For modeling purposes, the criterion for successful weight loss was broken down into discrete portions for each time interval, resulting in monthly $\alpha$ being set to 0.4 . For $\beta$, comparable models used in marketing applications of social networks were considered. In these models, the forgetfulness concept is reflected by a decay factor that depends on the characteristics of the population being investigated. For this simulation, $\beta$ was set at 0.1 , meaning that $90 \%$ of the effect received in previous step is remembered, carrying over to the next time interval. With $\delta$, the value was set to 0.3 , indicating that $70 \%$ of the ability to lose weight is saved and $30 \%$ is lost after each time interval. This value was selected based on the literature on the model of weight loss ability over time [20]. A worst case value was selected to better represent a realistic condition. Table 1 summarizes the values assigned 
Table 1. Values assigned to the model constants and variables for simulation purpose.

\begin{tabular}{|c|c|c|c|}
\hline Variable & Concept & Value & Reference \\
\hline$\alpha$ & Target percentage of weight loss & $5 \%$ a year & Blackburn [30] \\
\hline$\beta$ & Forgetfulness constant & 0.1 & $\begin{array}{l}\text { Assumption based on similar } \\
\text { diffusion models for } \\
\text { marketing purpose }\end{array}$ \\
\hline$\delta$ & $\begin{array}{c}\text { The decrease in ability to lose } \\
\text { weight }\end{array}$ & 0.3 & $\begin{array}{l}\text { Assumption based on the } \\
\text { model by Kevin Hall[21] }\end{array}$ \\
\hline Height & Each node's height in centimeters & $\mathrm{N}(169.35,84.70)$ & ) Ogden et al. [33] \\
\hline BMI & Each node's body mass index & $\mathrm{N}(35.9,13.61)$ & Flegal et al. [34] \\
\hline Willingness & $\begin{array}{l}\text { Initial willingness of nodes to take } \\
\text { part in a weight loss program }\end{array}$ & $\mathrm{U}(0,1)$ & Assumption \\
\hline Threshold & $\begin{array}{l}\text { Initial threshold of nodes to either } \\
\text { accept or refuse effectiveness of } \\
\text { the program }\end{array}$ & $\mathrm{U}(0,1)$ & Assumption \\
\hline Ability & $\begin{array}{l}\text { Initial ability of each node to lose } \\
\text { weight during a year }\end{array}$ & $\mathrm{N}(4,5.1)$ & Womble et al. [35] \\
\hline
\end{tabular}

to the model's constants and variables for simulation purpose. The simulation was run 1000 times, each time assigning random values of height, BMI, willingness, threshold and ability to the nodes, based on the reported population distributions, clinical trial results, or literature-supported assumptions (see Table 1).

\subsection{Simulation Results and Discussion}

Figure 2 shows the results for total weight loss of all six scenarios and the average of multiple runs of a random selection scenario. In addition, a summary of the total weight loss and number of successful participants after a 12 month period is shown in Table 2 . A one-way analysis of variance (ANOVA) was used to test for total network weight loss differences among the scenarios. The mean total weight loss in the network significantly differed among tested scenarios (main effect $p<0.001$ ). Post-hoc comparisons using Tukey's HSD indicated that the mean total weight loss did not differ significantly among individual-based scenarios, but the weight loss for these three scenarios was significantly larger than that of network-based scenarios (see Figure 3). As the results indicate, choosing the $10 \%$ of nodes with the highest ability outperforms the other scenarios. Since the outcomes of the three individual-based scenarios are not significantly different (see figure 2), selecting the nodes with the highest BMI as initial seeds may be most applicable, as BMI data are easily attainable. Our findings suggest that despite the importance of an individual's network-related attributes, their personal traits are of primary importance when targeting the intervention. In practice, due to lack of information available about the network structure, network-based scenarios may be infeasible. The findings of this model provide practitioners with support for networkbased interventions that account for personal attributes. Our findings are consistent with 


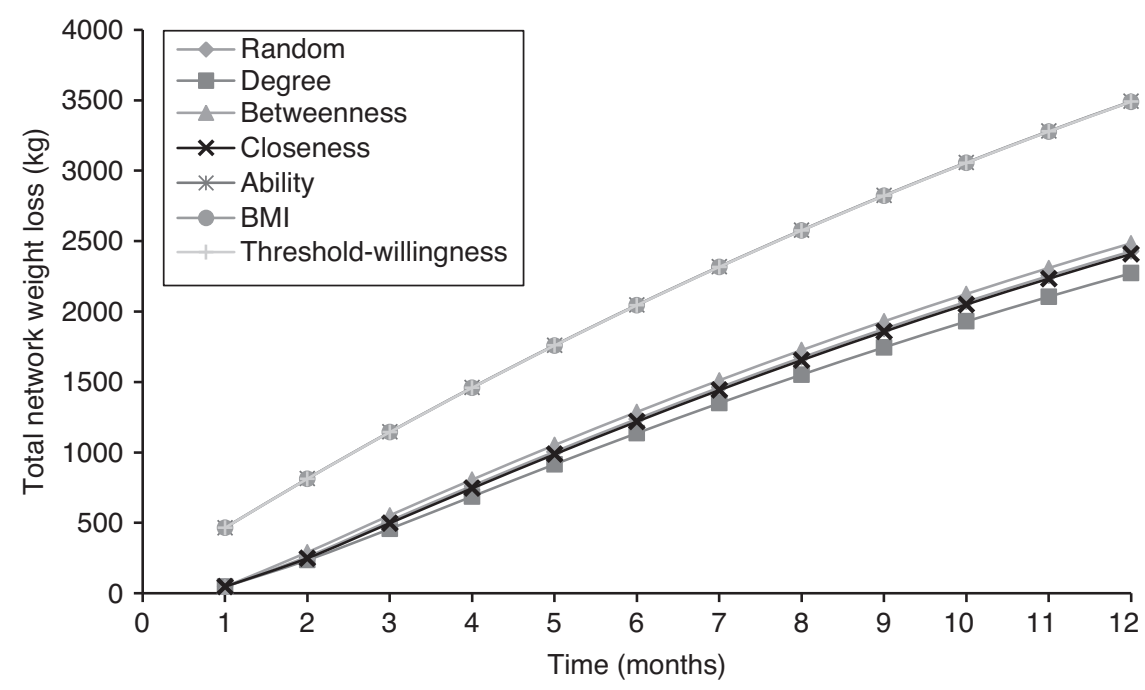

Figure 2. Total network weight loss for six different scenarios and random selection of initial seeds.

Table 2. Results by initial seed selection scenario, in a network of 1034 nodes, for all three objective functions considered.

\begin{tabular}{lccc}
\hline Scenario & $\begin{array}{c}\text { Number of } \\
\text { successful nodes }\end{array}$ & $\begin{array}{c}\text { Weight loss per } \\
\text { participant (kg) }\end{array}$ & $\begin{array}{c}\text { Total network } \\
\text { weight loss (kg) }\end{array}$ \\
\hline Highest degree & $189.7(32.5)$ & $3.23(0.15)$ & $2429.9(283.6)$ \\
Highest betweenness centrality & $207.8(21.5)$ & $3.30(0.14)$ & $2651.2(175.7)$ \\
Highest closeness centrality & $204.0(22.0)$ & $3.26(0.14)$ & $2573.6(191.6)$ \\
Highest ability to lose weight & $284.6(15.9)$ & $3.57(0.12)$ & $3692.1(139.0)$ \\
Highest BMI & $283.8(15.5)$ & $3.57(0.12)$ & $3688.8(139.7)$ \\
Highest initial value of threshold & & & \\
$\quad$ - willingness & $284.0(16.1)$ & $3.57(0.12)$ & $3689.4(147.4)$ \\
Random selection & $308.0(24.8)$ & $3.27(0.14)$ & $2593.1(198.5)$ \\
\hline
\end{tabular}

the findings of El-Sayed et al. [28], suggesting that selection of initial seeds based on their degree in the network is not the best strategy; however, as our findings suggest, selection scenarios based on individual-based attributes such as BMI can lead to better efficacy of network-based interventions. Clustering of individuals based on body status was a primary assumption for our model, taking into consideration the reported homophily in a network based on body weight.

\subsection{Sensitivity Analysis of Model Constants}

The model contains three basic constants either derived from the literature or set as program goals. In order to test the model's behavior across different values of these 


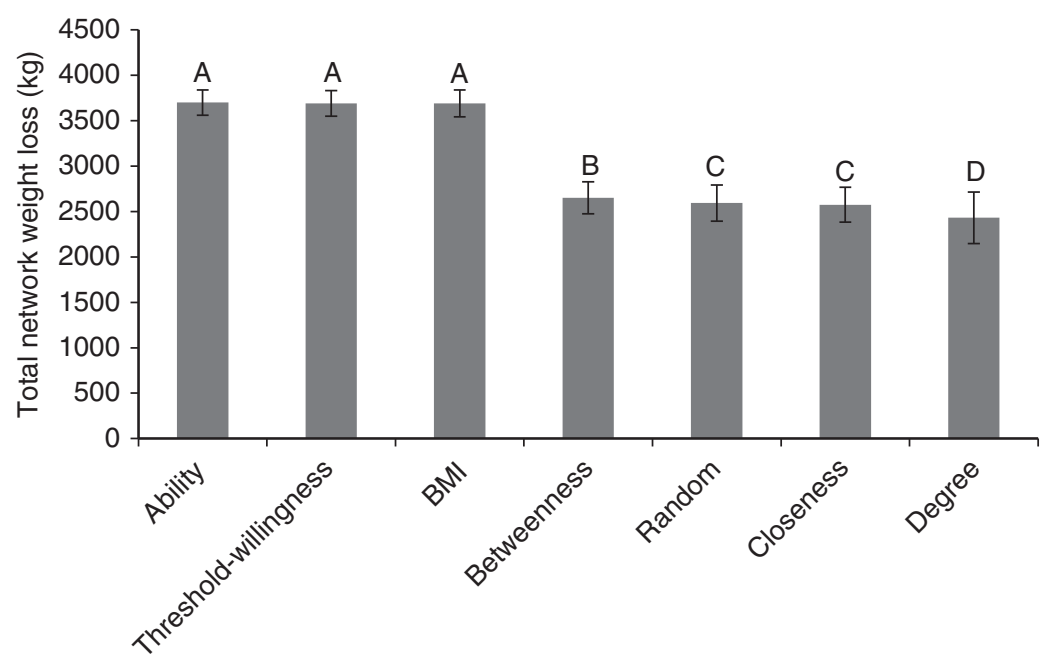

Figure 3. Total network weight loss for six different scenarios and random selection of initial seeds (Letter above each bar indicate results from the post-hoc comparison).

constants, the simulation was run 1000 times, each across different values of these constants. Theoretically, $\alpha$ can be set at different points in the range of 0 to $100 \%$ (0 to 1.0). It is the proportion of weight loss that is considered as successful in short time intervals. Setting it at 0 means the program keeps everyone in the network until the end, and no matter how much weight someone loses, he/she keeps sending out effect to the neighbors. In reality, when not successful enough, individuals will cease to participate due to lack of perceived positive outcome. As shown in Figure 4, the model behavior was tested over a range of $\alpha$ value. By increasing $\alpha$, meaning to be more restrictive of whom to consider successful, the total weight loss and weight loss per participating node decrease due to an increase in the number of dismissed nodes and an increase in the effect each activated node can generate. The $\alpha$ value can be set by the intervention administrators based on their goals and the intervention's target population. Across each of the scenarios available for selecting the initial seeds, the model behavior remained the same; therefore, only the results from selecting the seeds randomly are shown.

In order to model natural forgetfulness, $\beta$ represents the decay in memory allowing an individual to best remember the most effect from the most immediately previous time period. Ranging from 0 to 1 , a $\beta$ value equal to 0 means no forgetfulness effect exists and nodes remember all previously received effects equivalently. $\beta=1$ means that nodes forget all previously received effects. As the concept of $\beta$ implies, we expected to see that total weight loss decreases as $\beta$ goes up, since having more forgetful nodes decreases the accumulated effect and thus decreases the likelihood of surpassing the threshold to engage. These results are shown in Figure 5.

Generally speaking, as time goes by, a participating individual's ability to lose weight declines $[13,20,21]$. We considered this by decreasing the ability value with a constant 


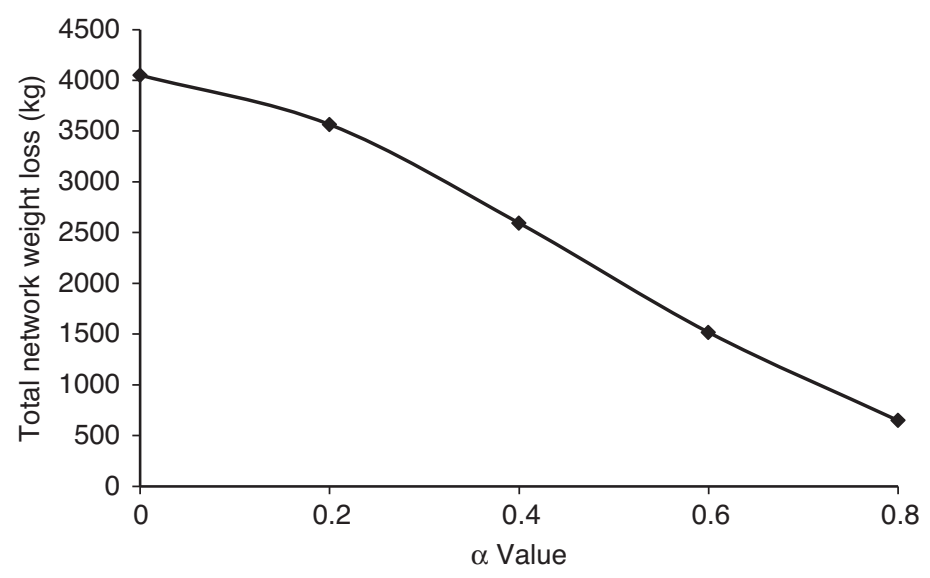

Figure 4. Changes in total network weight loss across different $\alpha$ values with randomly selected seeds.

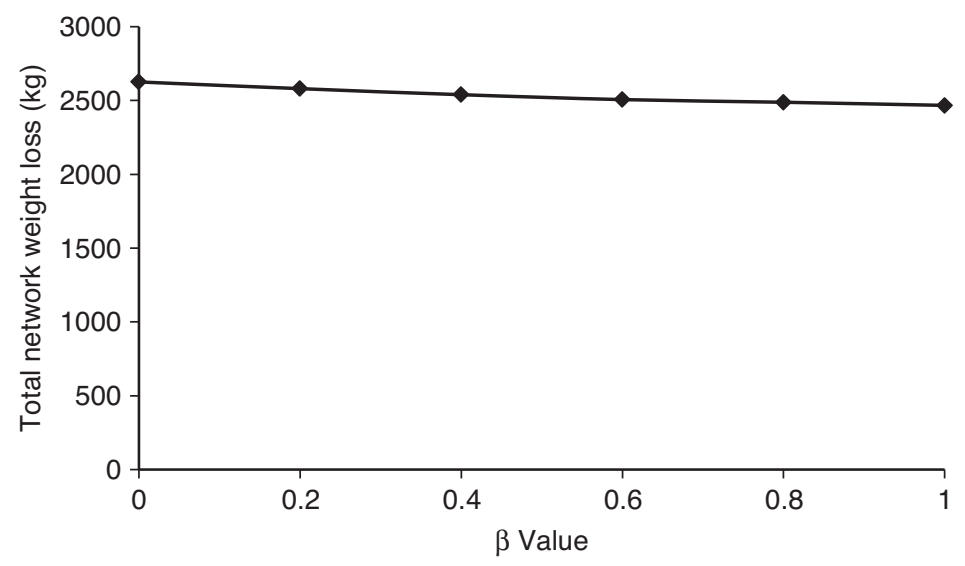

Figure 5. Changes in total network weight loss across different $\beta$ values with randomly selected seeds.

rate of $\delta$ ranging from 0 (the node loses no ability over time) to 1 (the node loses all of its ability). It was expected that an increase in $\delta$ would be accompanied by a steep decline in total weight loss and weight loss per participating node, primarily because a decrease in ability is equivalent to a decrease in the effect an activated node can generate and propagate throughout the network. Secondarily because, for every activated node, his/her own weight loss rate decreases and less weight is lost by already activated nodes. Total network weight loss over a range of $\delta$ values is shown in Figure 6. 


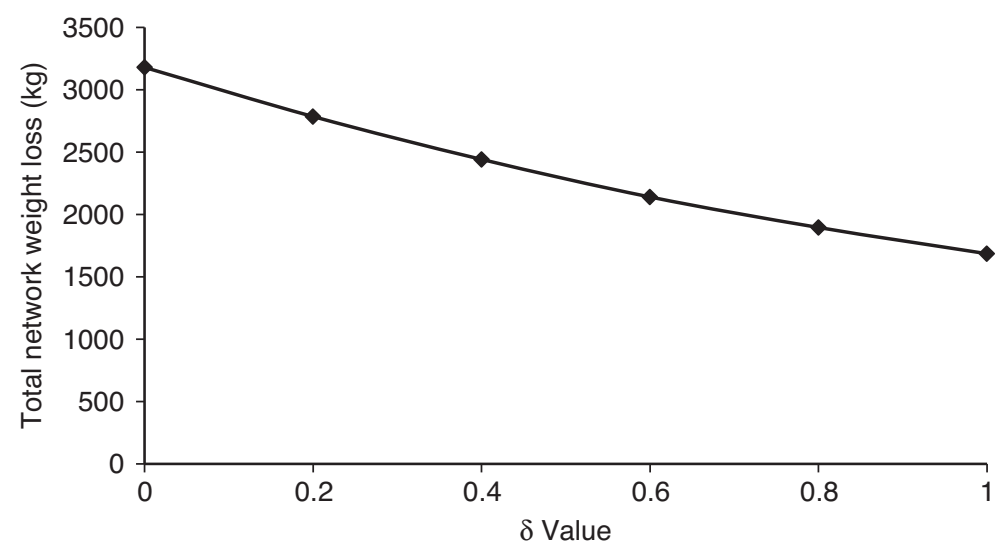

Figure 6. Changes in total network weight loss across different $\delta$ values with randomly selected seeds.

\subsection{Effects of Changing Network Density}

To understand the influence of network topology on diffusion of the weight loss effect, the random scenario was tested on a similar network with reduced densities. Random elimination of edges with a probability of $p$ was performed to cut down the number of edges and the network density. The program diffusion over 12 months was simulated using this reduced density network, and the results were compared to the original network (see Figure 7). As expected, due to the altered propagation of the weight loss effect, the total network weight loss decreases as the network density declines. These findings are consistent with that of Giabbanelli et al. [15], since the total network

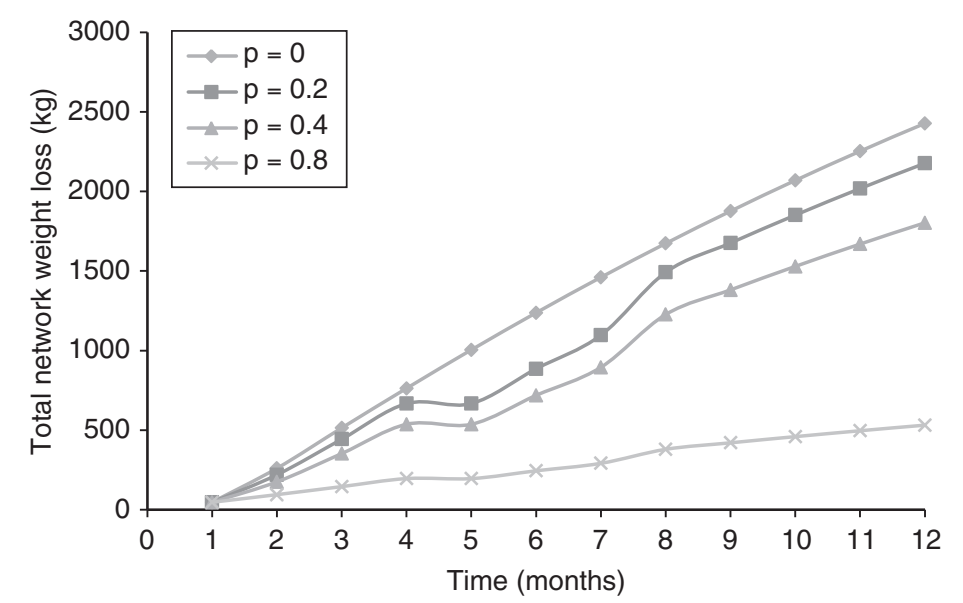

Figure 7. Total network weight loss for random scenario of choosing subset of initial seeds in networks of different density. 
weight loss significantly drops as nodes become less connected $(p<0.001$, based on a one-way ANOVA), meaning that the network structure contributes to the efficacy of the intervention.

\subsection{Applying the Greedy Algorithm}

In order to understand the goodness of solutions for the proposed diffusion model, it was compared versus an established algorithm. If we set the decay factor to 0 and consider a fixed weight loss over each time interval for all active nodes, then the model reduces to Linear Threshold model as introduced by the pioneering work of Kempe et al. [15]. The Linear Threshold model is also equivalent to Independent Cascade which holds the submodularity property. In solving influence maximization problem in social networks, the greedy algorithm, although not computationally efficient, guarantees the maximum gap to the optimal solution by providing a bound around the optimal solution. This algorithm provides a $\left(1-\frac{1}{e}\right)$ approximation for the optimal solution where $e$ is the natural logarithm. In order to employ the greedy algorithm, we need to test all single nodes as single seeds in the network and calculate the contribution as the total weight loss in the network for all single nodes. In the next step, the node with the maximum contribution will be selected as a seed and the process continues for finding the next single seed while the previous seed is already selected. This process continues until $k$ seeds are selected. The pseudo code for the greedy algorithm is provided in Table 3. Greedy algorithm has been customized for the proposed diffusion model presented in this paper. The results of this computation are illustrated in Figure 9. This method

Table 3. Pseudocode for the greedy algorithm.

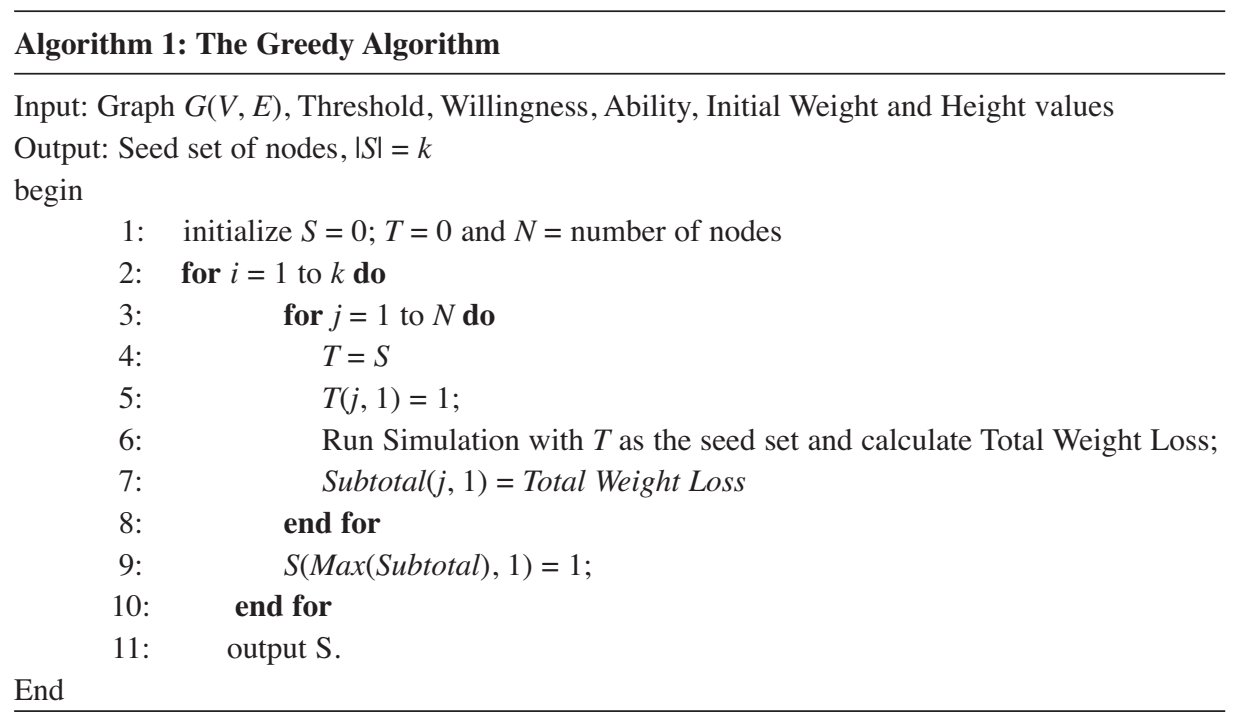




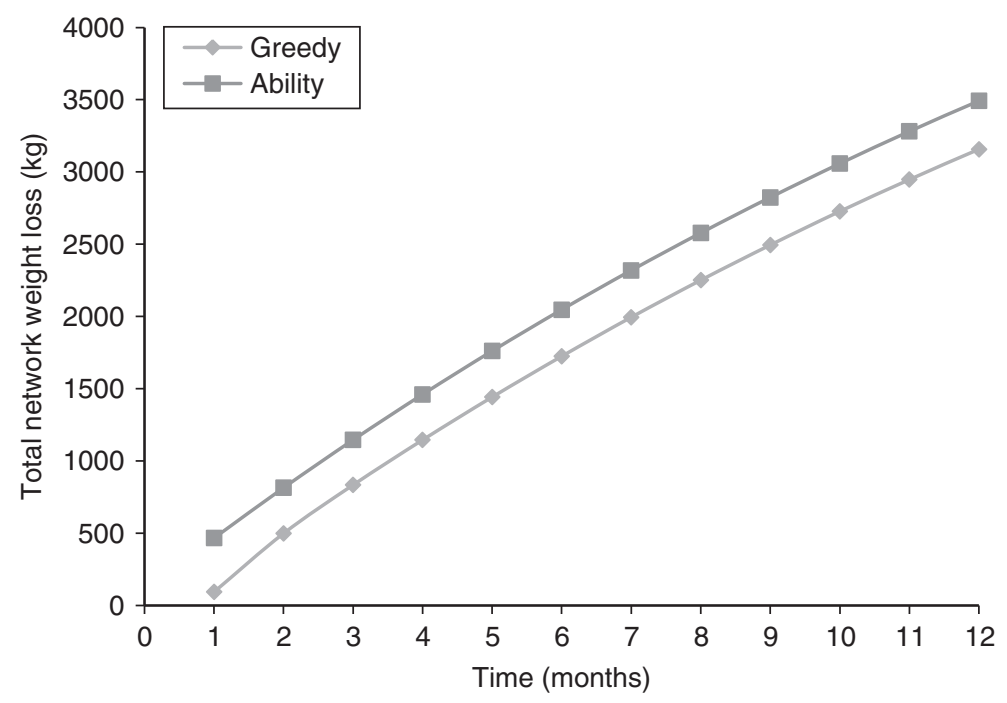

Figure 9. Total network weight loss for greedy algorithm versus ability scenario for selecting initial seeds.

required 25.5 hours of computation time compared to 6 seconds to run the model based on selecting the nodes with the highest ability. Since greedy algorithm guarantees the goodness of solution to be at most $\left(1-\frac{1}{e}\right)$ percent far from the optimal, under the tested condition, individual-based scenarios' outcome can be guaranteed to have smaller gap from the optimum solution. These results indicate that reasonable program success can be obtained based on our heuristic method.

\subsection{Study Limitations}

In considering the findings of the current work, there are several limitations to consider. First, while we attempted to incorporate the most important factors relevant to the success of a weight loss program, there could be other factors that affect intervention spread that were not tested here. External effects from individuals outside the network were assumed to have no impact on nodes, in comparison to the effects perceived from their peers, present in the network. Natural changes in the network population have also been excluded, which may impact the topology of the network over time. Future work could consider additional factors that may be relevant for program design.

Second, in testing the proposed model, we used network data extracted from an online social network, which might have different characteristics from offline networks. This also led to the assumption that the relationship between all nodes was friendship. Due to this, the specific findings may not generalize to other networks of varying sizes, arrangements, and relationship types. 
Third, each of the nodes was assigned randomly generated values of initial ability, threshold, and willingness. Random values were used due to a lack of available data for defining specific values. The parameters for defining the random values were informed based on the available literature, providing a reasonable estimate for the values; however, further research should be conducted to determine equations or models of these parameters for application in future social network models.

Fourth, verification of such models has not been completed, yet it is possible through longitudinal studies. In order to do so, two social networks of potential participants with a similar structure should be considered. Initial seeds should then be targeted and the changes in the network weight should be tracked for a year period. Comparison between the outcomes of different scenarios for selecting initial seeds can serve as a verification strategy for this model. Diffusion models are applied in other domains, specifically marketing and have been shown to have positive outcomes; nevertheless their application in health domain needs further investigation. Despite these limitations, our findings have important implications for understanding the spread of weight loss interventions.

\subsection{Discussion}

Our findings suggest that despite the importance of an individual's network-related attributes, their personal traits are of primary importance. Weight loss behavior is not a one-time decision and has at least two phases of initiation and continuation. The proposed framework takes into account the most important characteristics of a weight loss intervention and tries to model it. Still, future work needs to be done in order to quantify willingness and threshold values that we introduced. Individuals may experience a barrier to entering a weight loss program, which is reflected as the threshold in the model. Meanwhile, willingness toward complete participation in the program is represented as well. It is shown that an individual's ability to lose weight is dependent on many factors; high novelty seeking is reported to be associated with less success in losing weight [25]. Extracting the real-world network data is cumbersome, however possible. Engagement of people in prominent online social networks provides a chance for public health administers to take advantage of this platform. As we propose, Facebook data can be used as a source to extract network related attributes, especially in younger populations. Running surveys with standard scales can serve as a strategy to collect personal traits, such as willingness to lose weight or the ability to do so. Future studies can take advantage of ability scale introduced by Dash and Brown [36] in order to quantify an individual's ability.

\section{CONCLUSIONS}

The existence of peer effect on weight loss has been studied. Of concern is answering the question of whether peers influence each other to adapt a positive health-related behavior. While previous findings support the spread of positive behavior through large social networks, there is still place for assessing through known techniques in social network analysis that can be applied in order to enhance efficacy of network-based interventions. The current work provides a framework to consider both personal and 
social network aspects of weight loss interventions among connected individuals. This framework can direct weight loss intervention administers to identify individuals who can better propagate the weight loss effect in a large social network. There remains a need to assess the factors that can influence the spread of a network-based intervention. Large-scale health-related interventions are complex systems and modeling has the potential to improve these programs for considerable public health benefit.

\section{CONFLICT OF INTEREST}

The authors certify that they have no affiliations with or involvement in any organization with any financial interest or non-financial interest in the subject matter or materials discussed in this manuscript.

\section{NOMENCLATURE}

Variables:

$B_{i t}=$ Ability of node $\mathrm{i}$ to lose weight between time $\mathrm{t}_{-1}$ and $\mathrm{t}$ while $\mathrm{B}_{\mathrm{i} 0}$ is its initial ability $L_{i t}=$ Willingness of node $\mathrm{i}$ to take part in a weight loss program while $\mathrm{Li}_{0}$ is its initial willingness

$W_{i t}=\mathrm{B}$ ody mass of node at time $\mathrm{t}$ while $\mathrm{W}_{\mathrm{i} 0}$ is its initial body mass

$\sigma_{i}=$ Threshold of node $\mathrm{i}$ for either accepting or rejecting the effectiveness of the program

Constants:

$\alpha=$ Target percentage of weight loss based on what shown to have beneficial health effect

$\beta=$ Forgetfulness constant, hypothetically can range from 0 to 1

$\delta=$ Rate of decrease in ability

\section{REFERENCES}

[1] Fakhouri THI ,Ogden CL, Carroll MD, Kit BK, Flegal KM. Prevalence of Obesity Among Older Adults in the United States, 2007-2010. US Department of Health and Human Services, Centers for Disease Control and Prevention, National Center for Health Statistics, 2012.

[2] Ogden CL, Carroll MD, Kit BK, Flegal KM. Prevalence of obesity in the United States, 2009-2010. US Department of Health and Human Services, Centers for Disease Control and Prevention, National Center for Health Statistics, 2012.

[3] Mokdad AH, Bowman BA, Dietz WH, Vinicor F, Bales VS, Marks JS. Prevalence of obesity, diabetes, and obesity-related health risk factors, Journal of the American Medical Association, 2003, 289(1):76-79.

[4] Finkelstein EA, Fiebelkorn IC,Wang G. National Medical Spending Attributable To Overweight And Obesity: How Much, And Who's Paying?, Health Affairs, 2003

[5] Sturm R, The effects of obesity, smoking, and drinking on medical problems and costs. Health Affairs, 2002, 21(2):245-253.

[6] Cunningham SA, Vaquera E, Maturo CC, Narayan KMV. Is there evidence that friends influence body weight? A systematic review of empirical research. Social Science \& Medicine, 2012, 75: 1175-1183.

[7] Leahey TM, Kumar R, Weinberg BM, Wing RR. Teammates and social influence affect weight loss outcomes in a team-based weight loss competition. Obesity, 2012, 20:1413-1418.

[8] Rosenquist JN, Murabito J, Fowler JH., Christakis NA. The spread of alcohol consumption behavior in a large social network. Annals of Internal Medicine, 2010, 152(7):426-W141.

[9] Leahey TM, Crane MM, Pinto AM, Weinberg B, Kumar R, Wing RR.Effect of teammates on changes in physical activity in a statewide campaign. Preventive Medicine. 2010, 51(1):45-49. 
[10] Gesell SB, Tesdahl E, Ruchman E. The distribution of physical activity in an after-school friendship network, Pediatrics, 2012, 129(6):1064-1071.

[11] Shoham DA, Tong L, Lamberson PJ, Auchincloss AH, Zhang J, Dugas L Luke A. An actor-based model of social network influence on adolescent body size, screen time, and playing sports. PloS one, 2012, 7(6):e39795.

[12] Christakis NA, Fowler JH. The spread of obesity in a large social network over 32 years, New England Journal of Medicine, 2007, 357:370-9.

[13] Sentacik T, Atanasijeviæ-Kunc M, Drinovec J, Pfeifer M. Efficacy analysis of a body-mass-reduction treatment using mathematical modelling, Mathematical and Computer Modelling of Dynamical Systems: Methods, Tools and Applications in Engineering and Related Sciences, Mathematical and Computer Modelling of Dynamical Systems, 2013.

[14] Leahey TM, LaRose JG, Fava JL, Wing RR. Social Influences Are Associated With BMI and Weight Loss Intentions in Young Adults, Obesity, 2011, 19:1157-1162.

[15] Giabbanelli PJ, Jackson PJ, Finegood DT. Modelling the joint effect of social determinants and peers on obesity among Canadian adults. Theories and Simulations of Complex Social Systems. Springer Berlin Heidelberg, 2014, 145-160.

[16] Bahr DB, Browning RC, Wyatt HR, Hill JO. Exploiting social networks to mitigate the obesity epidemic. Obesity, 2009, 17:723-728.

[17] Kempe D, Kleinberg J, Tardos B. Maximizing the spread of influence through a social network. In Proceedings of the ninth ACM SIGKDD international conference on Knowledge discovery and data mining, ACM, 2003, 137-146.

[18] Sciamanna CN, Tate DF, Lang W, Wing RR. Who reports receiving advice to lose weight? Results from a multistate survey. Archieves of Internal Medicine. 2000, 160(15):2334-2339.

[19] Goldstein DJ. Beneficial health effects of modest weight loss. International Journal of Obesity and Related Metabolic, 1992, 16(6):397-415.

[20] Hall KD, Sacks G, Chandramohan D, Chow CC, Wang YC, Gortmaker SL, Swinburn BA. Quantification of the effect of energy imbalance on bodyweight, Lancet, 2011, 378:826-37.

[21] Hall KD. Computational model of in vivo human energy metabolism during semi-starvation and refeeding. American Journal of Physiology. Endocrinology and metabolism, 2006, 291.1:E23.

[22] Cogan JC , Rothblum ED. Outcomes of weight-loss programs. Genetic, Social, and General Psychology Monographs, 1992, 118(4):385-415.

[23] Appel LJ, Clark JM, Yeh HC, Wang NY, Coughlin JW., Daumit G, Miller ER, Dalcin A, Jerome GJ, Geller S, Noronha G, Pozefsky T, Charleston J, Reynolds JB, , Durkin N, Rubin RR, Louis TA, Brancati FL. Comparative effectiveness of weight-loss interventions in clinical practice.New England Journal of Medicine, 2011, 365(21):1959-68.

[24] Subramani MR, Rajagopalan B. Knowledge-sharing and influence in online social networks via viral marketing, Communications of the ACM - Mobile computing opportunities and challenges, 2003, 64(12):300-307.

[25] Sullivan S,Cloninger CR, Przybeck TR, Klein S. Personality characteristics in obesity and relationship with successful weight loss. International Journal of Obesity, 2007, 31:669-674.

[26] Giabbanelli PJ, Alimadadb A, Dabbaghianb V, Finegooda DT. Modeling the influence of social networks and environment on energy balance and obesity. Journal of Computational Science, 2012(3):17- 27.

[27] Rozin P, Royzman EB. Negativity bias, negativity dominance, and contagion. Personality and Social Psychology Review, 2001, 5(4):296-320.

[28] El-Sayed AM, Seemann L, Scarborough P, Galea S. Are Network-Based Interventions a Useful Antiobesity Strategy? An Application of Simulation Models for Causal Inference in Epidemiology, American Journal of Epidemiology, 2013, 178(2). 
[29] Leskovec J. Stanford large network dataset collection, 2011, [Facebook Data]. Retrieved from http://snap.stanford.edu/data/egonets-Facebook.html.

[30] Kanders BS., Blackburn GL. Wadden TA. (Ed); VanItallie TB. (Ed). Reducing primary risk factors by therapeutic weight loss. Treatment of the seriously obese patient, Guilford Press, New York, NY, US, 1992, 213-230.

[31] Blackburn G. Effect of degree of weight loss on health benefits. Obesity Research, 1995, 3(2):211-216.

[32] Tsai AG, Wadden TA. Systematic review: an evaluation of major commercial weight loss programs in the United States. Annals of Internal Medicine, 2005, 142.1, 56-66.

[33] Ogden CL. Mean body weight, height, and body mass index: United States 1960-2002. Department of Health and Human Services, Centers for Disease Control and Prevention, National Center for Health Statistics, 2004.

[34] Flegal KM, Carroll MD, Kit BK, Ogden CL, Prevalence of obesity and trends in the distribution of body mass index among US adults, 1999-2010, 2012, Journal of the American Medical Association, 307.5 (2012):491-497.

[35] Womble LG, Wadden TA, McGuckin BG, Sargent SL, Rothman RA, Krauthamer-Ewing ES .A randomized controlled trial of a commercial internet weight loss program. 2004, Obesity Research, 12(6):1011-1018.

[36] Dash JD, Brown RA. The development of a rating scale for the prediction of success in weight reduction, Journal of Clinical Psychology, 1977, SS(3). 


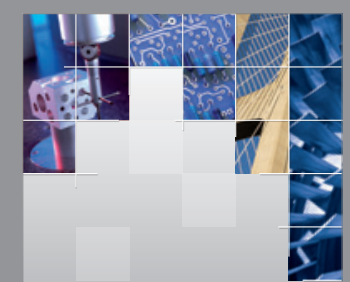

\section{Enfincering}
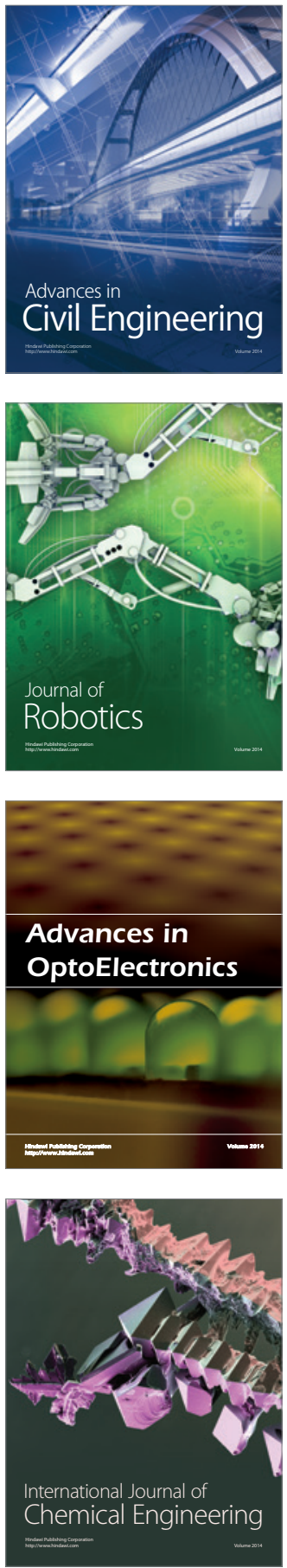

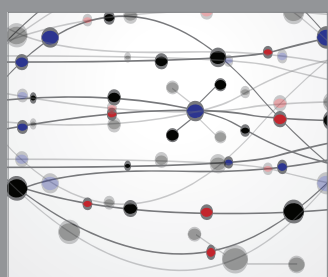

The Scientific World Journal

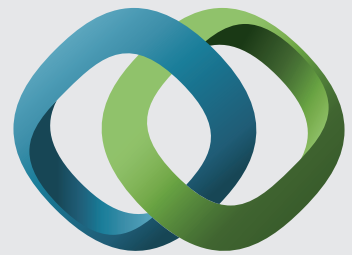

\section{Hindawi}

Submit your manuscripts at

http://www.hindawi.com
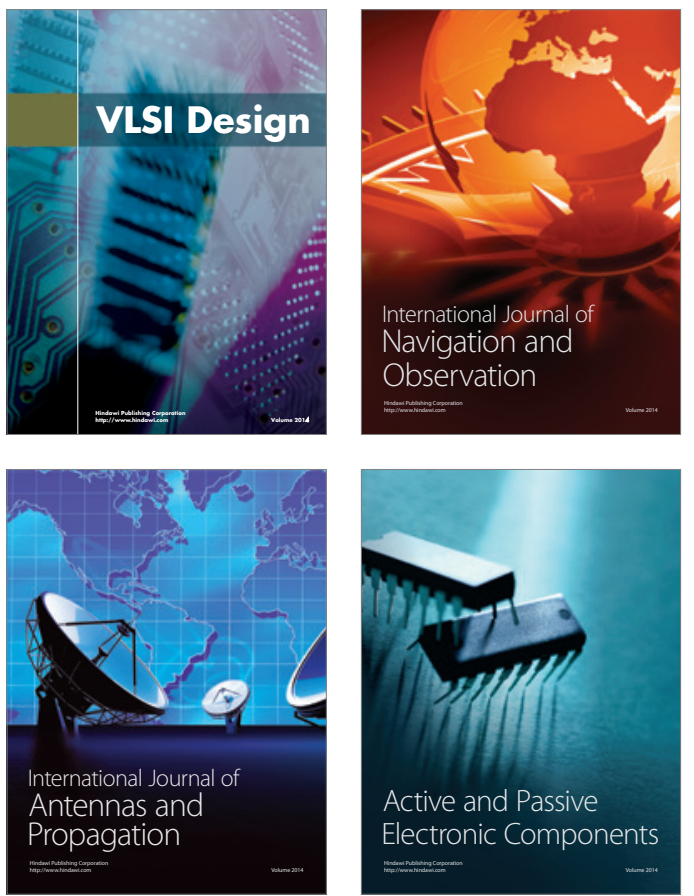
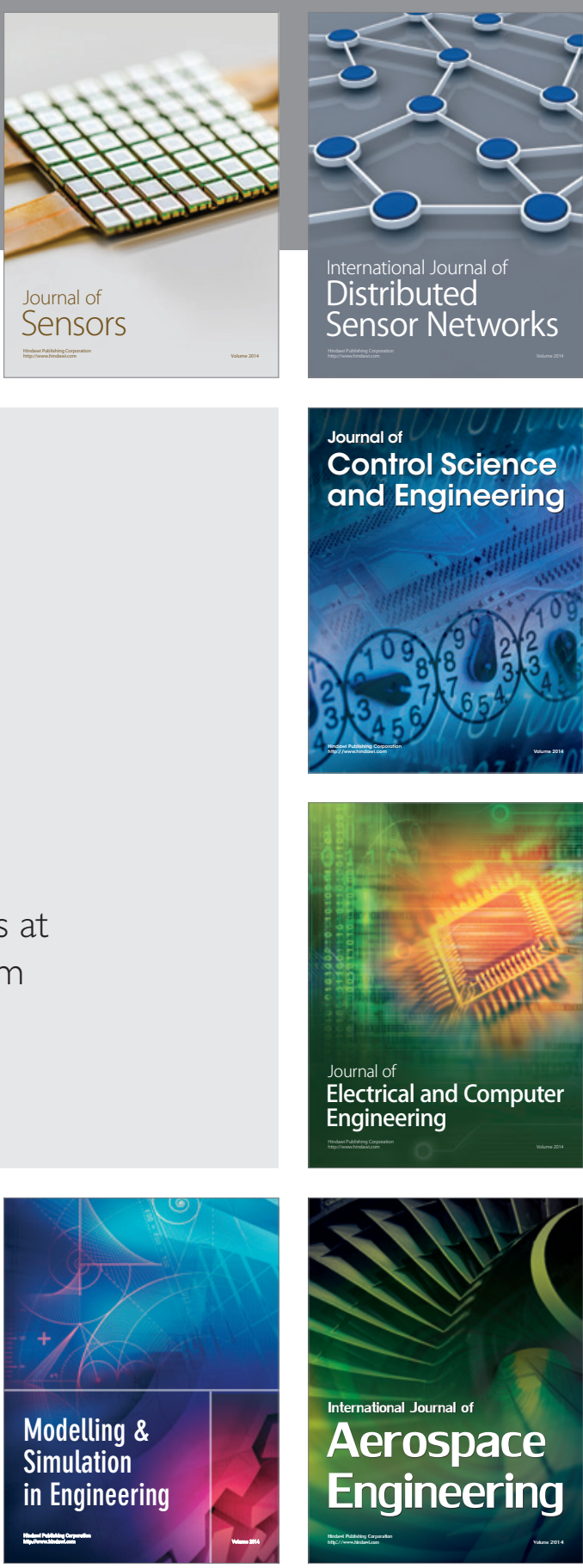

International Journal of

Distributed

Sensor Networks

Journal of

Control Science

and Engineering
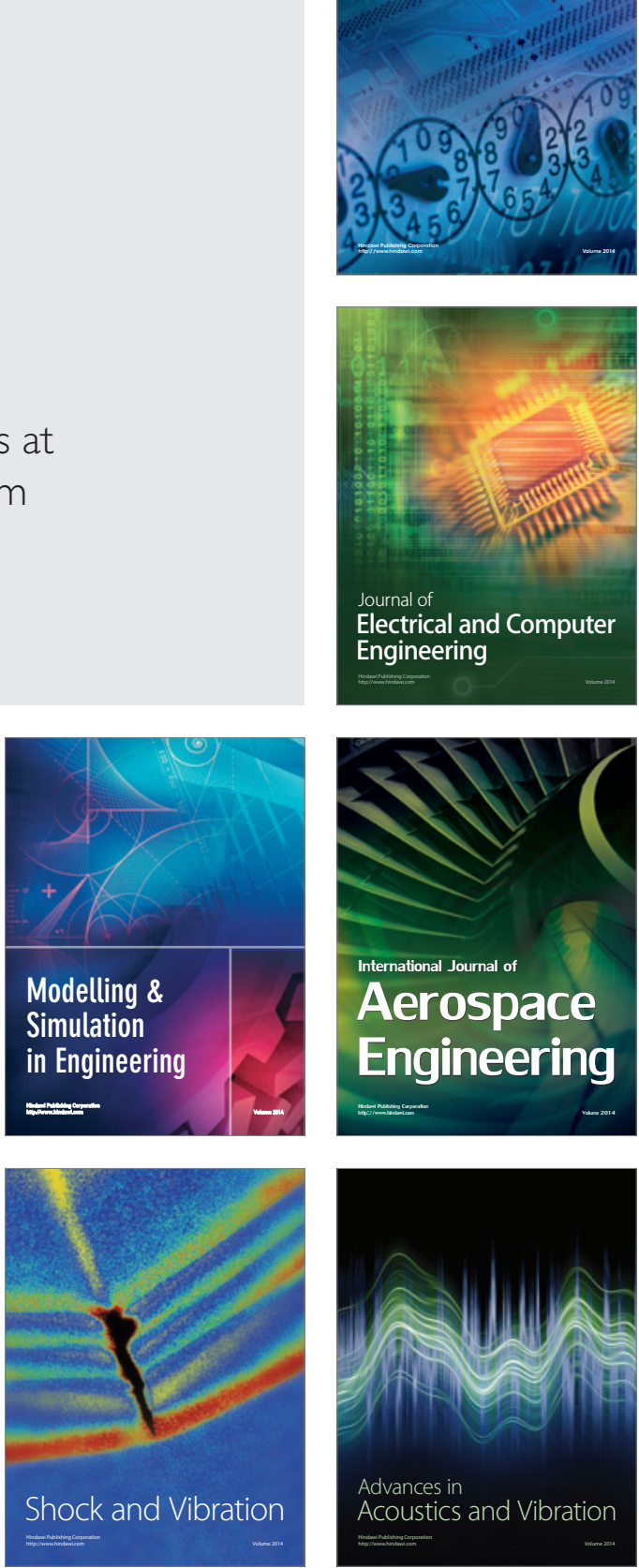